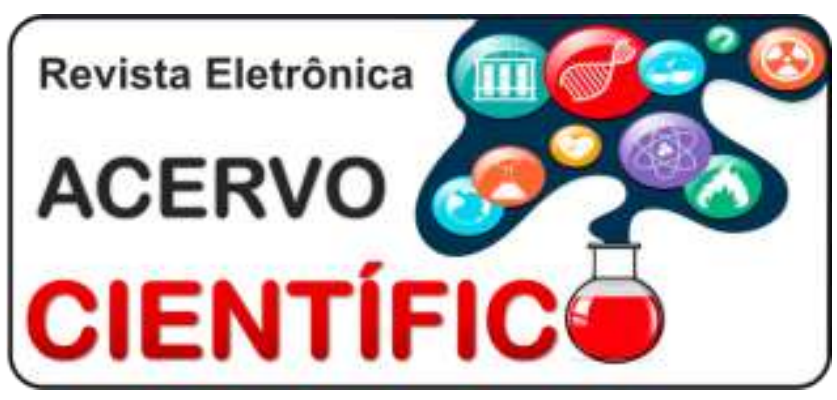

\section{REVISÃO BIBLIOGRÁFICA}

Recebido em: 9/2019

Aceito em: 10/2019

Publicado em: 12/2019

\title{
Fatores de risco associados a sepse neonatal: Artigo de revisão
}

\author{
Risk factors related with neonatal sepsis: Review article
}

Factores de riesgo relacionados con la sepsis neonatal: Artículo de revisión

Ana Rosa Felizola Dortas ${ }^{1 *}$, David Martins da Silva Mello ${ }^{1}$, Lucas Alves Bezerra ${ }^{1}$, Ricardo Gois de Lima ${ }^{1}$, Victoria Haydée Deusdedith Neves ${ }^{1}$, José Aderval Aragão'.

Resumo: Este artigo buscou analisar os principais fatores de risco para desenvolvimento da sepse neonatal. O estudo é uma revisão da literatura atual e foram incluídos na pesquisa 17 artigos da plataforma PUBMED com os descritores: sepse neonatal, infecção, fatores de risco. De forma geral os fatores mais prevalentes encontrados na literatura atual foram sexo masculino, muito baixo peso ao nascer, nascidos pré-termo, Apgar menor ou igual 3 no 5ำ minuto, uso de métodos invasivos de tratamento ou monitorização, ruptura prematura ou prolongada de membranas, deficiência de glicose-6-fosfato desidrogenase e infecção do trato urinário da mãe pelos agentes etiológicos. Conclui-se que a sepse neonatal é uma afecção decorrente da interação de múltiplos fatores de risco, tanto maternos quanto fetais, e se torna de suma importância reconhecê-los a fim de prevenir o desenvolvimento da sepse neonatal e proporcionar intervenções mais precoces.

Palavras-chave: Sepse neonatal, Fatores de risco, Infecção.

\begin{abstract}
This article proposes to analyze the major risk factors for the development of neonatal sepsis. This study is a current literature review and were included 17 articles from PUBMED using the keywords: neonatal sepsis, infection, risk factors. Overall the most prevalent factors found in the literature were male newborn, very low birth weight, preterm labor, Apgar score lower or equal 3 in the $5^{\text {th }}$ minute, use of invasive treatment or monitorization methods, premature or prolonged membrane rupture, glucose-6-phosphate dehydrogenase deficiency and mother's urinary tract infection. To conclude, neonatal sepsis is an affection due to the interaction of multiple mother's and newborn's risk factors; so, it is of great importance to recognize in order to prevent its development and provide earlier interventions.
\end{abstract}

Keywords: Neonatal sepsis, Risk factors, Infection.

Resumen: Este artículo tuvo como objetivo analizar los principales factores de riesgo para el desarrollo de sepsis neonatal. El estudio es una revisión de la literatura actual y se incluyeron 17 artículos de la plataforma PUBMED en la investigación con los descriptores: sepsis neonatal, infección, factores de riesgo. En general, los factores más prevalentes encontrados en la literatura actual fueron el sexo masculino, muy bajo peso al nacer, nacimientos prematuros, Apgar menor o igual a 3 en el quinto minuto, uso de tratamiento invasivo o

${ }^{1}$ Universidade Tiradentes (UNIT), Aracaju-Sergipe. *E-mail: anarosafelizola@gmail.com 
métodos de monitoreo, ruptura prematura o prolongada de membranas, deficiencia de glucosa-6-fosfatodeshidrogenasa e infección del tracto urinario materno por agentes etiológicos. Se concluye que la sepsis neonatal es una condición resultante de la interacción de múltiples factores de riesgo, tanto maternos como fetales, y es extremadamente importante reconocerlos para prevenir el desarrollo de sepsis neonatal y proporcionar intervenciones más tempranas.

Palabras clave: Sepsis neonatal, Factores de riesgo, Infección.

\section{INTRODUÇÃO}

Sepse neonatal é a síndrome da resposta inflamatória sistêmica resultante da suspeita ou confirmação de infecção com ou sem bacteremia, documentada por uma cultura positiva nos 28 primeiros dias de vida. Pode ser classificada como de início precoce ou tardio. A sepse neonatal precoce aparece até o $7^{\circ}$ dia de vida, normalmente nas primeiras 24 horas de vida. Contudo em pré-termos aparece até o $3^{\circ}$ dia de vida. A sepse neonatal tardia ocorre após o $7^{\circ}$ dia de vida (HAMMAND E e ZAINAB MS, 2018).

A incidência da sepse neonatal é responsável pela ocorrência de cinco milhões de óbitos em RNs, (recémnascido), sendo os países subdesenvolvidos ou em desenvolvimento, como o Brasil, sede da maioria dos casos diagnosticados. Os grupos mais susceptíveis são os recém-nascidos (RN) de baixo peso submetidos a procedimentos invasivos durante o período de internação na Unidade de Terapia Intensiva Neonatal (UTIN) (OLIVEIRA COP, et al., 2016).

Os fatores de risco associados ao desenvolvimento de tal condição clínica podem ser estratificados com base na classificação da sepse neonatal em precoce e tardia. Na sepse de início precoce a principais condições de risco para seu desenvolvimento são maternas, como colonização por Streptococcus do grupo $\mathrm{B}$ (como o pyogenes), corioamnionite, ruptura prematura de membranas, ruptura prolongada de membranas (maior que 18 horas), infecção do trato urinário materno, múltiplas gestações e parto pré-termo (CAMACHOGONZALEZ A, et al., 2013).

A sepse neonatal tardia, por sua vez, possui os principais fatores de risco de origem do RN, como quebra de barreiras naturais (pele e mucosas), uso prolongado de cateteres de permanência, execução de procedimentos invasivos (intubação endotraqueal, por exemplo), enterocolite necrosante e uso prolongado de antibióticos, bloqueadores de receptores histamínicos $\mathrm{H} 2 \mathrm{e}$ inibidores da bomba de prótons. $\mathrm{O}$ baixo peso ao nascer (menor que 1500 gramas) e idade gestacional menor que 37 semanas são condições de risco para ambas formas de sepse neonatal. (CAMACHO-GONZALEZ A, et al., 2013).

Esta revisão bibliográfica é justificada pela alta incidência de sepse neonatal, mesmo com a evolução tecnológica que viabiliza seu diagnóstico, e por ainda ser considerada um grande problema de saúde pública no Brasil e no mundo. O principal objetivo da revisão literária foi identificar principais fatores de risco para desenvolvimento de sepse neonatal.

\section{MÉTODOS}

O presente artigo é uma revisão integrativa da literatura com análise e síntese dos resultados observados. A questão que guiou esta revisão foi "Quais são os principais fatores de risco para o desenvolvimento de sepse neonatal?".

Foi realizada uma busca através da base de dados do Medical Literature Analysis and Retrieval System Online (MEDLINE) por meio do mecanismo de busca Public/ Publisher MEDLINE. Cada descritor de interesse foi procurado usando a ferramenta Medical Subject Headings (MeSH). Os descritores utilizados foram: sepse neonatal, infecção e fatores de risco os quais foram combinados na seguinte estratégia de busca: "Neonatal Sepsis"[Mesh]) AND "Risk Factors"[Mesh] AND "infection". Esses descritores foram selecionados devido ao foco em publicações relacionadas às características do objeto de estudo. 
Foram utilizados os seguintes critérios de inclusão: artigos na base de dados selecionada em português ou inglês, publicados nos últimos 5 anos. Critérios de exclusão foram artigos com texto incompleto e revisões literárias.

A busca foi realizada pelo acesso online aos artigos relevantes nos meses de agosto e setembro de 2019. Foi feita uma análise do material selecionado para reunir informações relevantes buscando uma melhor compreensão do tema.

\section{RESULTADOS E DISCUSSÃO}

Nesta revisão integrativa foram incluídos 14 artigos. Foram encontrados 45 artigos no PubMed. Destes, 28 foram excluídos por não atenderem os critérios propostos. Tais artigos estão dispostos na tabela 01.

O estudo observacional e prospectivo de Softić I, et al. (2017) teve como amostra 200 neonatos admitidos na Unidade de Terapia Intensiva Neonatal (UTIN), dos quais 136 tiveram pelo menos um episódio de sepse neonatal. Constatou-se que a incidência de sepse precoce foi de $28,7 \%$, enquanto a da tardia, de $71,3 \%$. Ainda acerca de tal dado, faz-se importante ressaltar que houve maior taxa de sepse tardia entre os neonatos de muito baixo peso (inferior a 1500 gramas).

Ocviyanti D e Wahono W (2018) mostraram que a incidência de sepse neonatal comprovada por hemoculturas positivas foi de 5,2\%. Além disso, verificou-se que o peso médio ao nascer de bebês com sepse neonatal foi de 1.420 gramas, enquanto para aqueles sem sepse neonatal foi de 2.560 gramas.

Esse estudo de Ocviyanti D e Wahono W (2018) mostrou que os recém-nascidos (RN) com sepse neonatal também tiveram maior tempo de permanência no hospital, com média de 32 dias. Além da relação com a idade gestacional da mãe, a sepse neonatal foi encontrada em apenas 1 paciente $(0,5 \%)$ no parto a termo em comparação com 20 pacientes $(9,1 \%)$ no parto pré-termo.

Freitas F e Romero G (2016) analisaram dados de 2014 a 2015 e, nesse período, um total de 36 neonatos foram testados positivos para sepse neonatal dentre os 13,627 nascidos vivos no hospital estudado. $O$ Streptococcus do grupo B foi o agente mais isolado em todas a culturas (19,4\%), seguido por E. coli (13,8\%).

Todos os 36 pacientes da pesquisa de Freitas F e Romero G (2016) desenvolveram quadro sintomático. Um fato associado ao surgimento do quadro foi a via de parto. $53 \%$ nasceram por parto vaginal e $50 \%$ das mães sofrem ruptura precoce da bolsa amniótica, fatores estes associados com as maiores taxas de infecção nesses neonatos.

A análise realizada por Wu I-H, et al. (2017) constatou 948 casos de infecção da corrente sanguínea (ICS) em 732 neonatos, correspondendo a uma incidência de 3,71 casos a cada 1 mil pacientes de tal faixa etária. Foi observado que, dentre estes casos, 781 foram resultados de bacteremia primária, enquanto os outros $17,6 \%$ possuíam algum foco específico de infecção, sendo os mais comuns a meningite, a pneumonia associada à ventilação mecânica (PAV), a enterocolite necrosante e a infecção sanguínea relacionada a cateter central.

O estudo feito por Wu I-H, et al. (2017) ainda afirma que a microbiologia da sepse neonatal varia de acordo com o foco infeccioso, de forma que na bacteremia primária, os agentes etiológicos mais comuns são os Staphylococcus coagulase-negativos; na meningite, o Streptococcus do grupo B; na PAV, os bacilos Gramnegativos e na infecção sanguínea associada à cateter central, os patógenos Gram-positivos.

Ademais Wu I-H, et al. (2017) demonstram que as manifestações clínicas na bacteremia primária e da infecção sanguínea associada à meningite a ao cateter venoso central (CVC) são similares, enquanto o quadro clínico daquelas associadas à enterocolite necrosante e à PAV são mais graves, com possível evolução para choque séptico, coagulação intravascular disseminada (CIVD), trombocitopenia, anemia e acidose metabólica. 
Tabela 1 - Principais fatores de risco encontrados nos estudos pesquisados.

\begin{tabular}{|c|c|c|c|c|}
\hline Autor & Revista/Jornal & Objetivo de Estudo & Fatores de Risco e Resultados & $\%$ \\
\hline $\begin{array}{l}\text { Alcock G, et al. } \\
\qquad(2017)\end{array}$ & BMC Pediatrics & $\begin{array}{l}\text { Comparar um conjunto de medidas para o cuidado de um acesso } \\
\text { para nutriçăo parenteral, utilizando técnica asséptica rigorosa com } \\
\text { um acesso que recebeu cuidados normais e associá-los à } \\
\text { ocorrência de sepse neonatal de inicio tardio. }\end{array}$ & $\begin{array}{l}\text { - Não houve diferença significativa de casos } \\
\text { de sepse entre as crianças dos grupos da } \\
\text { técnica asséptica e da técnica padrão. } \\
\text { - Para bebês }<1000 \mathrm{~g} \text { também não revelaram } \\
\text { diferença na taxa de sepse entre os grupos } \\
\text { intervenção e controle. }\end{array}$ & - \\
\hline $\begin{array}{l}\text { Alves JB, et al. } \\
\qquad(2018)\end{array}$ & Revista Paulista de Pediatria & $\begin{array}{l}\text { Descrever o coeficiente de mortalidade neonatal por sepse e outras } \\
\text { causas, além das caracteristicas maternas, gestacionais, do parto, } \\
\text { do recém-nascido e do óbito em Londrina, Paraná. }\end{array}$ & $\begin{array}{l}\text { - Pré-eclâmpsia } \\
\text { - Infecção materna do trato urinário } \\
\text { - Indice Apgar < } 7 \text { no primeiro e quinto minutos }\end{array}$ & $\begin{array}{l}- \\
-\end{array}$ \\
\hline $\begin{array}{l}\text { Cekmez Y, et al. } \\
\qquad(2017)\end{array}$ & Ginekolgia Polska & $\begin{array}{l}\text { Avaliar o papel do volume plaquetário médio para predição pré-natal } \\
\text { de sepse neonatal de início precoce em bebês nascidos a termo de } \\
\text { mães com baixo risco de infecção. }\end{array}$ & $\begin{array}{l}\text { - Alto volume plaquetário médio materno - } \\
\text { grupo estudado vs. grupo controle }(8,27 \\
\pm 1.85 \text { vs. } 8.98 \pm 1.16 ; p=0.001)\end{array}$ & \\
\hline $\begin{array}{l}\text { Freitas FT e } \\
\text { Romero GA } \\
\text { (2017) }\end{array}$ & $\begin{array}{l}\text { Brazilian Journal of } \\
\text { Infectious Diseases }\end{array}$ & $\begin{array}{l}\text { Descrever a epidemiologia de sepse neonatal precoce em uma } \\
\text { maternidade pública de Brasilia, Brasil. }\end{array}$ & $\begin{array}{l}\text { - Colonização materna por Streptococcus do } \\
\text { grupo B }\end{array}$ & $24 \%$ \\
\hline $\begin{array}{l}\text { Gebremedhin D. } \\
\text { Et al. (2016) }\end{array}$ & PLoS One Journal & $\begin{array}{l}\text { Estudo baseado em controle de casos realizados em hospitais } \\
\text { públicos da cidade de Mekelle, região de Tigray no norte da Etiópia. } \\
\text { Foram incluidos } 78 \text { casos de neotanos que tiveram sepse e } 156 \\
\text { casos controle de neonatos que năo tinham sepse. Foram avaliados } \\
\text { os possiveis fatores de risco implicados na sepse neonatal. }\end{array}$ & $\begin{array}{l}\text { - História de infecção do trato urinário ou } \\
\text { infecçōes sexualmente transmissiveis. } \\
\text { - Ruptura prolongada de membranas } \\
\text { - Parto hospitalar } \\
\text { - Febre no intraparto } \\
\text { - APGAR }<7 \text { no } 5^{\circ} \text { minuto } \\
\text { - Não chorar imediatamente após o } \\
\text { nascimento }\end{array}$ & - \\
\hline $\begin{array}{l}\text { Heo JS. et al. } \\
\text { (2018) }\end{array}$ & Pediatrics \& Neonatolgy & $\begin{array}{l}\text { Avaliar os fatores de risco para anormalidades moderadas a graves } \\
\text { da substância branca do cérebro em bebês extremamente } \\
\text { prematuros (idade gestacional }<28 \text { semanas) com sepse neonatal. }\end{array}$ & $\begin{array}{l}\text { - Idade gestacional média de } 25 \text { semanas } \\
\text { - Peso médio ao nascer de } 727 \text { gramas } \\
\text { Baixo escore Apgar no primeiro e quinto } \\
\text { minutos } \\
\text { - Sexo masculino }\end{array}$ & - \\
\hline $\begin{array}{l}\text { Kung Y. et al. } \\
\text { (2016) }\end{array}$ & $\begin{array}{l}\text { Journal of microbiology, } \\
\text { immunology, and infection }\end{array}$ & $\begin{array}{l}\text { Avaliar registros das infecçōes da corrente sanguinea de julho de } \\
2003 \text { a junho de } 2006 \text { em uma unidade de terapia intensiva neonatal } \\
\text { em Taiwan. Avaliando-se peso ao nascer, idade gestacional, sexo, } \\
\text { indice de Apgar e data de nascimento. }\end{array}$ & $\begin{array}{l}\text { - Idade gestacional média } \\
\text { - } \quad \text { Peso ao nascer } \\
\text { - } \quad \text { Staphylococos coagulase-negativos } \\
\text { - } \quad \text { Klebsiella pneumoniae }(14,6 \%) \\
\text { - } \quad \text { Candida spp. }(6,7 \%) \\
\text { - } \quad \text { Hemoçāo parenteral } \\
\text { - Hemogia intraventricular }\end{array}$ & $\begin{array}{c}30,7 \pm 0,7 \\
1512 \pm 804 \mathrm{~g} \\
(28,7 \%) \\
(16,5 \%) \\
(14,6 \%) \\
(6,7 \%) \\
-\end{array}$ \\
\hline
\end{tabular}




\begin{tabular}{|c|c|c|c|c|}
\hline $\begin{array}{l}\text { Mahallei M, et al. } \\
\qquad(2018)\end{array}$ & Medicine & $\begin{array}{l}\text { Analisar os resultados das culturas de sangue, líquido } \\
\text { cefalorraquidiano e urina nos casos suspeitos de sepse neonatal no } \\
\text { nordeste do Irã }\end{array}$ & $\begin{array}{l}\text { - } \quad \text { Hospitalização prévia } \\
\text { - Cirurgias }\end{array}$ & $\begin{array}{l}40,19 \% \\
21,56 \%\end{array}$ \\
\hline $\begin{array}{l}\text { Moges F. et al. } \\
\text { (2017) }\end{array}$ & BMC Pediatrics & $\begin{array}{l}\text { Estudo transversal realizado em neonatos com evidencias clínicas } \\
\text { de Sepse na University of Gondar Hospital de Setembro/2015 até } \\
\text { Maio/2016. Foram incluídos na pesquisa } 251 \text { neonatos. Verificou- } \\
\text { se os patógenos bacterianos e suas susceptibilidades por meio de } \\
\text { métodos microbiológicos padronizados. }\end{array}$ & $\begin{array}{l}\text { - } 117 \text { apresentaram crescimento bacteriano } \\
\text { - } 49 \text { foram infectados por Staphylococcus } \\
\text { aureus } \\
\text { - } 25 \text { infectados por Sthaphylococcus } \\
\text { coagulasse-negativos } \\
\text { - } 18 \text { por Klebsiella pneumoniae }\end{array}$ & $\begin{array}{l}46,6 \% \\
41 \% \\
21 \%\end{array}$ \\
\hline & & & & $15 \%$ \\
\hline $\begin{array}{l}\text { Ocviyanti D e } \\
\text { Wahono W } \\
(2018)\end{array}$ & Journal of pregnancy & $\begin{array}{l}\text { Estudo transversal no Hospital Cipto Mangunkusumo, Jacarta, de } \\
\text { dezembro de } 2016 \text { a junho de } 2017.0 \text { mesmo utilizou o método de } \\
\text { amostragem total, incluindo todas as mulheres grávidas com idade } \\
\text { gestacional de } 20 \text { semanas ou mais com ruptura prematura da } \\
\text { membrana, que chegaram ao hospital na época. }\end{array}$ & $\begin{array}{l}\text { - Hemoculturas positivas } \\
\text { - } \quad \text { Peso médio reduzido ao nascer } \\
\text { - } \quad \text { Sepse no pormanência hospitalar } \\
\text { - }\end{array}$ & $\begin{array}{l}5,2 \% \\
- \\
32 \text { dias } \\
1 \times 20\end{array}$ \\
\hline $\begin{array}{l}\text { Rostamir Far Z. } \\
\text { Et al. (2016) }\end{array}$ & J Med Life & $\begin{array}{l}\text { Estudo realizado de Fevereiro/2012 até Novembro/2014 na região } \\
\text { leste do Iran. Foram incluidos } 76 \text { neonatos com sepse e } 1214 \\
\text { neonatos sem sepse. Foi avaliado o estado de deficiência de } \\
\text { glicose-6-fosfato desidrogenase e comparado entre os integrantes } \\
\text { do estudo. Concluiu-se que a deficiência de G6PD é um fator de } \\
\text { risco para sepse neonatal. }\end{array}$ & $\begin{array}{l}\text { - Maior prevalência de deficiência de G6PD } \\
\text { em neonatos com sepse }(p=0.03)\end{array}$ & - \\
\hline Reed et al. (2018) & The Journal of Pediatrics & $\begin{array}{l}\text { Examinar o impacto da exposição pré-natal a antibióticos maternos } \\
\text { no risco de enterocolite necrosante, sepse tardia) e morte em bebês } \\
\text { nascidos prematuros. }\end{array}$ & $\begin{array}{l}\text { - Exposição materna a antibióticos até } 72 \\
\text { horas antes do parto }\end{array}$ & - \\
\hline $\begin{array}{l}\text { Softić l, et al. } \\
\text { (2017) }\end{array}$ & Acta Medica Academica & $\begin{array}{l}\text { Avaliar a incidência, mortalidade, fatores de risco, etiologia e } \\
\text { susceptibilidade aos antibióticos das bactérias responsáveis por } \\
\text { sepse }\end{array}$ & $\begin{array}{l}\text { - Muito baixo peso ao nascer } \\
\text { - } \quad \text { Prematuridade extrema (<28 semanas) } \\
\text { - Escore de Apgar no } 5^{\circ} \text { minuto } \leq 3 \\
\text { - } \quad \text { Ventilação assistida } \\
\text { - } \quad \text { Interença de CVC Ções cirúrgicas }\end{array}$ & $\begin{array}{l}- \\
- \\
- \\
-\end{array}$ \\
\hline $\begin{array}{l}\text { Wu I, et. al. } \\
\quad(2017)\end{array}$ & BMC Infectious Diseases & $\begin{array}{l}\text { Determinar os fatores de risco, microbiologia e implicaçōes clínicas } \\
\text { da infecção de corrente sanguínea neonatal com um foco } \\
\text { concomitante de infeç̧ão }\end{array}$ & $\begin{array}{l}\text { - } \quad \text { Hipertensão pulmonar secundária } \\
\text { - } \quad \text { Presença de complicações infecciosas } \\
\text { - História de um ou mais episódios prévios de } \\
\quad \text { infecção de corrente sanguinea neonatal }\end{array}$ & $\begin{array}{l}- \\
- \\
- \\
-\end{array}$ \\
\hline
\end{tabular}

Fonte: Dortas AR, et al., 2019.

REAC/EJSC | Vol. 7 | e1861 | DOI: https://doi.org/10.25248/reac.e1861.2019 Página $\mathbf{5}$ de $\mathbf{8}$ 
O estudo descritivo-analítico de Mahalley M, et al. (2018) teve como amostra de estudo todos os neonatos com menos de um mês de idade que se encontravam internados na UTIN ou na ala pediátrica do hospitalsede da pesquisa e que apresentavam manifestações clínicas sugestivas de sepse. Foram constatados 838 casos suspeitos, dos quais $102(12,17 \%)$ tiveram culturas positivas, preenchendo, assim, os critérios para diagnóstico de sepse. Nessa pesquisa por Mahalley M, et al. (2018), observou-se que a incidência de sepse confirmada em relação ao total de neonatos hospitalizados foi de $3,87 \%$, sendo mais frequente entre os pacientes do sexo masculino $(59,8 \%)$. Tal estudo ainda demonstrou que a média da idade gestacional dos neonatos foi de 36,91 semanas, sendo $76,47 \%$ destes nascidos a termo, e que a idade média para 0 aparecimento sepse neonatal foi de 7 dias.

Outrossim, Mahalley M, et al. (2018) constataram que 48,23\% dos neonatos não possuíam fatores de risco para sepse e, nos pacientes nos quais estes foram notados, os mais comuns foram história de hospitalização prévia e cirurgias. Em relação aos patógenos envolvidos, percebeu-se que as bactérias mais comuns na hemocultura foram o Staphylococcus coagulase-negativo e o Staphylococcus aureus. Neste estudo, a mortalidade da sepse neonatal por Klebsiella pneumoniae, Acinetobacter e Enterobacter foi de 100\%.

Corroborando com esse dado, Moges F, et al. (2017) evidenciaram que dos 251 casos suspeitos de sepse neonatal, $67,5 \%$ apresentaram cultura com bactérias Gram-positivas. Sendo as mais comuns $S$. Aureus $(40,8 \%)$, seguido por Staphylococcus coagulase-negativo $(21,6 \%)$ e K. pneumoniae (15,8\%). Além desses achados, foi percebido que das cepas Gram-positivas e negativas isoladas $70 \%$ foram organismos multirresistentes às drogas.

De acordo com os achados de Kung Y, et al. (2016), os Staphylococcus coagulase-negativos foram os organismos mais comuns responsáveis pela sepse neonatal na UTIN analisada.

Ainda de acordo com Kung Y, et al. (2016), sugeriu-se que o uso do CVC não aumentou substancialmente a incidência de ICS quando os prematuros apresentaram demografia e condição de saúde semelhantes ao nascimento. Em complemento, o uso pré-natal de esteróides não foi estatisticamente significativo na análise dos fatores de risco das infecções da corrente sanguínea neonatal, mesmo com maior incidência de ICS em bebês expostos ao tratamento com esteróides antes do nascimento.

Alcock G, et al. (2017) constataram a relação de nutrição parenteral (NP) com a sepse de início tardio. Não como entidade causadora, mas como um marcador da vulnerabilidade desses bebês. Visto que RN's que recebem NP tendem a ter procedimentos mais invasivos, como colocação de cateteres intravasculares e coleta de sangue.

Cekmez Y, et al. (2017) fizeram uma associação entre o volume plaquetário médio (VPM) materno e o desenvolvimento de sepse neonatal precoce. O estudo, que analisou 130 mulheres não trombofílicas, concluiu que a dosagem do VPM é um método de baixo custo, não invasivo e clinicamente significativo para predizer a chance de desenvolvimento de sepse neonatal, sendo níveis elevados mais associados com a doença.

Softić I, et al. (2017) ainda definiram os fatores de risco associados ao maior risco de desenvolvimento de sepse neonatal tardia: muito baixo peso ao nascer, prematuridade extrema (menor que 28 semanas), escore de Apgar do 5 minuto menor ou igual a três, ventilação assistida, presença de CVC e intervenções cirúrgicas.

A pesquisa supracitada feita por Softić I, et al. (2017) ainda demonstrou que a duração média de internação foi equivalente a 13,23 dias e que mortalidade por sepse neonatal correspondeu a 12,5\%, contribuindo, assim, para $89,3 \%$ dos óbitos no período neonatal.

Heo JS, et al. (2017) estudaram a correlação entre sepse no período neonatal e alterações da substância branca de tais pacientes. Em seu estudo foram incluídos 72 pacientes, dos quais $63(87,5 \%)$ apresentaram nenhuma ou discretas alterações da substância branca comprovadas por Ressonância Nuclear Magnética (RNM). Os outros 9 (12,5\%) apresentaram alterações consideradas moderadas ou graves ao exame. Os fatores de risco mais associados a essas alterações foram idade gestacional média de 25 semanas, peso médio ao nascer de 727 gramas, e baixo escore Apgar no primeiro e quinto minuto de vida. O sexo masculino foi o mais afetado. 
Reed B, et al. (2018) encontraram em seu estudo que a exposição materna a antibióticos até 72 horas antes do parto aumenta em 1,6 vezes a chance de recém-nascidos prematuros (<32 semanas) apresentarem quadro de sepse de início tardio.

Além dos fatores elencados pelos estudos acima, Rostamir-Far Z, et al. (2016) verificaram em seu estudo uma correlação entre a deficiência glicose-6-fosfato desidrogenase (G6PD) e o desenvolvimento de sepse neonatal. De acordo com esse estudo a deficiência de G6PD seria um importante fator de risco no desenvolvimento da sepse e poderia explicar a maior prevalência em RN's do sexo masculino.

Em relação à mortalidade, WU $\mathrm{I}-\mathrm{H}$, et al. (2017) estabelecem alguns fatores de risco associados a tal desfecho, tais como hipertensão pulmonar secundária à displasia broncopulmonar grave, comorbidades neurológicas, presença de complicações infecciosas e história de um ou mais episódios prévios de ICS neonatal.

Em estudo realizado analisando as condições associadas à mortalidade neonatal, Alves JB, et al. (2018) observaram que a maioria dos aspectos relacionados ao desenvolvimento da sepse poderiam ser prevenidos e que um acompanhamento pré-natal insatisfatório pode aumentar em até 10 vezes sua ocorrência.

Grebremedhin D, et al. (2015) finaliza em seu estudo definindo que os principais fatores de risco encontrados para desenvolvimento da sepse neonatal foram história de infecção urinária ou infecções sexualmente transmissíveis, febre intraparto, parto hospitalar, Ruptura prolongada de membranas, APGAR menor que 7 no $5^{\circ}$ minuto e não chorar no momento imediato ao parto. De forma geral, observa-se concordância entre os autores sobre os principais fatores que implicam na sepse neonatal. Salvo algumas variações de prevalência entre os estudos.

\section{CONSIDERAÇÕES FINAIS}

A sepse neonatal é uma condição clínica comum e associada à alta morbimortalidade. Tal afecção é decorrente da interação complexa de fatores de risco maternos e neonatais. Com base na literatura, os agentes etiológicos mais relevantes foram $S$. aureus e coagulase-negativos, K. pneumoniae e Streptococcus do grupo B. Acerca dos fatores de risco, os mais associados foram: sexo masculino, muito baixo peso ao nascer, nascidos pré-termo, Apgar baixo, realização de procedimentos invasivos, ruptura prematura ou prolongada de membranas, deficiência de G6PD, infecção do trato urinário materno, pré-natal inadequado e hospitalização prévia. A partir dos dados colhidos, conclui-se que se faz o reconhecimento das condições predisponentes ao desenvolvimento da sepse neonatal, visando sua prevenção e intervenção precoce.

\section{REFERÊNCIAS}

1. ALCOCK G, et al. Prevention of neonatal late-onset sepsis: a randomised controlled trial. Bmc Pediatrics, 2017; 17(1): 1-98.

2. ALVES JB, et al. Neonatal sepsis: mortality in a municipality in southern Brazil, 2000 to 2013. Rev. paul. Pediatr, 2018; 36 (2): $132-140$.

3. CAMACHO-GONZALEZ A, et al. Neonatal Infectious Diseases. Pediatric Clinics of North America, 2013; 60(2): 367389.

4. CEKMEZ Y, et al. The utility of maternal mean platelet volume levels for early onset neonatal sepsis prediction of term infants. Ginekologia Polska, 2017; 88(6): 312-314.

5. FREITAS F, ROMERO G. Early-onset neonatal sepsis and the implementation of group $B$ streptococcus prophylaxis in a Brazilian maternity hospital: a descriptive study. Brazilian Journal of Infectious Diseases, 2017; 21(1): 92-97.

6. GEBREMEDHIN D, et al. Risk factors for neonatal sepsis in public hospitals of Mekelle City, North Ethiopia, 2015: unmatched case control study. PloS one, 2016; 11(5): e0154798.

7. HAMMAD E, MS Z. Meta-Analysis on Factors Influencing Early Onset Neonatal Sepsis. Sch J Appl Sci Res, 2018; 1(8): 20-22.

8. HEO JS, et al. Timing of sepsis is an important risk factor for white matter abnormality in extremely premature infants with sepsis. Pediatrics \& Neonatology, 2018; 59(1): 77-84. 
9. KUNG Y, et al. Risk factors of late-onset neonatal sepsis in Taiwan: A matched case-control study. Journal Of Microbiology, Immunology And Infection, 2016; 49(3): 430-435.

10. MAHALLEI M, et al. Clinical symptoms, laboratory, and microbial patterns of suspected neonatal sepsis cases in a children's referral hospital in northwestern Iran. Medicine, 2018; 97(25): e10630.

11. MOGES F, et al. Bacterial etiologic agents causing neonatal sepsis and associated risk factors in Gondar, Northwest Ethiopia. BMC pediatrics, 2017; 17(1): 137.

12. OCVIYANTI D, WAHONO W. Risk Factors for Neonatal Sepsis in Pregnant Women with Premature Rupture of the Membrane. Journal Of Pregnancy, 2018; 2018: 1-6.

13. OLIVEIRA COP, et al. Fatores de risco para sepse neonatal em unidade de terapia: estudo de evidência. Cogitare Enferm, 2016; 21(2): 1-9.

14. REED B, et al. The Impact of Maternal Antibiotics on Neonatal Disease. The Journal of Pediatrics, 2018; 197: 97103.e3.

15. ROSTAMIR-FAR Z, et al. Glucose-6-phosphate dehydrogenase deficiency (G6PD) as a risk factor of male neonatal sepsis. Journal of medicine and life, 2016; 9(1): 34 .

16. SOFTIĆ I, et al. Bacterial sepsis in neonates: Single centre study in a Neonatal intensive care unit in Bosnia and Herzegovina. Acta Medica Academica, 2017; 46(1): 7-15.

17. WU $\mathrm{I}-\mathrm{H}$, et al. Incidence, clinical features, and implications on outcomes of neonatal late-onset sepsis with concurrent infectious focus. Bmc Infectious Diseases, 2017; 17(1): 17-465. 